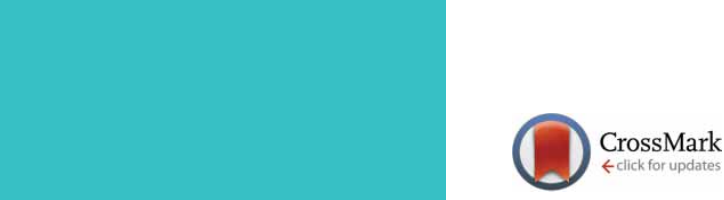

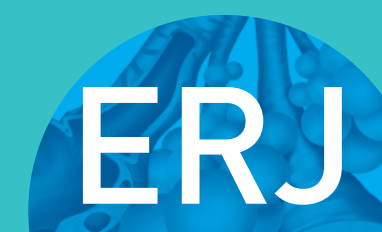

open research

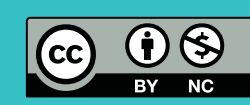

\section{Association of atypical antipsychotics and mortality for patients hospitalised with pneumonia}

\author{
Zachary Boivin ${ }^{1}$, Mario F. Perez ${ }^{1}$ Nkiruka C. Atuegwu', Mark Metersky ${ }^{1}$, \\ Carlos A. Alvarez ${ }^{2,3}$, Antonio Anzueto ${ }^{4,5}$ and Eric M. Mortensen ${ }^{1,2}$
}

Affiliations: ${ }^{1}$ University of Connecticut Medical Center, Farmington, CT, USA. ${ }^{2}$ VA North Texas Health Care System, Dallas, TX, USA. ${ }^{3}$ Texas Tech University Health Sciences Center, Dallas, TX, USA. ${ }^{4}$ South Texas Veterans Health Care System, San Antonio, TX, USA. ${ }^{5}$ University of Texas Health Science Center at San Antonio, San Antonio, TX, USA.

Correspondence: Eric Mortensen, UConn Health, 263 Farmington Avenue, Farmington, CT, 06030, USA. E-mail: mortensenduchc.edu

\section{ABSTRACT}

Introduction: Atypical antipsychotics are commonly used in patients with psychiatric conditions and dementia. They are also frequently used in patients being admitted with pneumonia; however, there are few safety data. The purpose of this study was to examine whether atypical antipsychotic use prior to admission is associated with increased mortality in patients with pneumonia.

Methods: We conducted a retrospective cohort study of hospitalised patients with pneumonia over a 10year period. We included patients 65 years or older and hospitalised with pneumonia. For our primary analysis, we used propensity score matching to balance confounders between atypical antipsychotic users and nonusers.

Results: There were 102897 patients and 5977 were taking atypical antipsychotics. After matching there were 5513 users and 5513 nonusers. Atypical antipsychotic use was associated with increased odds of 30day (OR 1.20, 95\% CI 1.11-1.31) and 90-day mortality (1.19, 1.09-1.30).

Conclusion: In patients 65 years or older that are hospitalised with pneumonia, we found an association between atypical antipsychotic use and increased odds of mortality. This was particularly pronounced for patients with pre-existing psychiatric or cardiac conditions. We suggest closely monitoring patients who use these medications and minimising their use in older adult patients.

@ERSpublications

When hospitalised with pneumonia, older patients who use atypical antipsychotics should be monitored closely and their use of these drugs should be minimised as much as possible http:// bit.ly/2JEevHV

Cite this article as: Boivin Z, Perez MF, Atuegwu NC, et al. Association of atypical antipsychotics and mortality for patients hospitalised with pneumonia. ERJ Open Res 2019; 5: 00223-2018 [https:// doi.org/10.1183/23120541.00223-2018].

Received: 23 Nov 2018 | Accepted after revision: 11 June 2019

Copyright $\odot$ ERS 2019. This article is open access and distributed under the terms of the Creative Commons Attribution Non-Commercial Licence 4.0. 


\section{Introduction}

Pneumonia continues to be a leading cause of death in the United States $[1,2]$. The vast majority of deaths due to pneumonia occur in patients over 65 years of age. In addition, this condition is responsible for a high financial burden with over US\$10 billion spent caring for patients with pneumonia [1].

Antipsychotics are used to treat psychiatric diseases such as psychosis, schizophrenia, and dementia, and are also used for the management of delirium and agitation. There are two categories of antipsychotic medications, typical and atypical antipsychotics. One of the main differences between the two groups is the safety profile. Typical antipsychotics have been shown to increase cardiac events, whereas atypical antipsychotics were designed to reduce that risk [3]. Although there have been studies demonstrating an association between the use of antipsychotics and increased mortality in older adult patients with dementia [4], there have been few studies that examine the association between typical antipsychotic medications and outcomes for patients with pneumonia [3-5]. One study examined the association of atypical antipsychotics with pneumonia and found no significant association with increased mortality [5]. Examining the safety of atypical antipsychotics will help physicians to make informed decisions about the continuation of atypical antipsychotic medications for patients hospitalised with pneumonia, who are at increased risk for cardiac events due to the infection as well as the antibiotics used [6-13].

The primary aim of this study was to examine the association between prior atypical antipsychotic use and 90-day all-cause mortality in patients 65 years and older that were hospitalised with pneumonia using data from the Department of Veterans Affairs (VA) Health Care System. Our a priori hypothesis was that being prescribed atypical antipsychotic medications would be associated with an increase in the odds of 90-day mortality in patients hospitalised with pneumonia.

\section{Materials and methods}

We conducted a retrospective cohort study using the clinical and administrative databases of the Department of VA Health Care System. These databases are the repositories of clinical data from all of the national VA hospitals and outpatient clinics [14]. This study was approved by the VA North Texas Institutional Review Board.

\section{Inclusion criteria}

We included patients who met the following criteria:

a) Hospitalisation between October 1, 2001 and September 30, 2012.

b) 65 years or older on the date of admission.

c) Discharged with a diagnosis of pneumonia defined as either a primary diagnosis of pneumonia (ICD-9 codes 480.0-483.99 or 485.0-487.0) or a secondary diagnosis of pneumonia with a primary diagnosis of respiratory failure (ICD-9 code 518.81) or sepsis (ICD-9 code $0.38 \mathrm{xx}$ ).

d) Had at least one dose of antimicrobial therapy within the first $48 \mathrm{~h}$ of admission.

e) Had at least three or more VA outpatient clinic visits in the year preceding admission.

f) Received at least one outpatient medication from a VA pharmacy within 90 days prior to admission, thereby ensuring that the patients were receiving medications from VA pharmacies.

We excluded those who received typical antipsychotics from further analyses due to these medications' clear associations with increased mortality. For patients who had multiple pneumonia-related hospitalisations during the study period, we included only their first hospitalisation.

\section{Data sources and definitions}

Atypical antipsychotics included risperidone, olanzapine, clozapine, quetiapine, ziprasidone, aripiprazole, amisulpride, paliperidone and sertindole $[4,5,15]$.

We controlled for race and ethnicity categories (white, black, and Hispanic), tobacco use (ICD-9 codes 305.1 and V15.82, smoking cessation clinic use, and/or use of medications for the treatment of nicotine dependence such as Zyban, nicotine replacement, or varenicline), alcohol abuse (ICD-9 codes 291, 303, 305.0), and illicit drug use (ICD-9 codes 292, 304, 305, excluding 305.0-0.1). We also used the Charlson-Deyo comorbidity system to identify pre-existing comorbid conditions [16], and the VA priority status for socioeconomic status [17]. Psychiatric conditions, including depression, bipolar disorder, post-traumatic brain disorder (PTSD) and schizophrenia, were identified using the method described by Selim et al. $[18,19]$.

Potential confounding medications were controlled for by using a count of unique drugs in each of the following classes for outpatient prescriptions filled within 90-days prior to presentation: antidepressants, lithium, statins, beta blockers, calcium channel blockers, oral antidiabetic agents, lipid-lowering agents, other antihypertensive agents, antiarrhythmic agents, inhaled beta agonists, other bronchodilators and theophylline. 


\section{Outcomes}

The primary outcomes of this study were all-cause mortality within 30- and 90-days of admission. Death was identified using the VA Vital Status file, which has an 98\% accuracy in identifying mortality [20].

\section{Statistical analysis}

For the primary analyses, we used propensity score matching to balance measured confounders between groups (atypical antipsychotic users versus nonusers). Logistic regression was used to create the propensity score and then nearest-number matching with a calliper of 0.001 with no replacement was performed [21]. We selected candidate variables that we believed would be potentially associated with severity of illness (e.g. intensive care unit admission, use of mechanical ventilation), outcomes (e.g. age, nursing home residence), or with prescription of antipsychotics (e.g. dementia, psychiatric conditions.) Variables included in the propensity score are displayed in table 1 . Odds ratios were calculated to determine the association between atypical antipsychotic use and the outcomes using conditional logistic regression.

To analyse the time-to-event for mortality and cardiovascular outcomes by receipt of antipsychotics, we used Kaplan-Meier plots to display the survivor functions and assessed statistical significance using the log rank test.

For secondary analyses, we used generalised linear mixed effect models to examine the association of atypical antipsychotic use on 90-day mortality in the following pre-defined subgroups: dementia, psychiatric conditions (PTSD, schizophrenia, bipolar disorder and depression), pre-existing cardiac conditions (myocardial infarction, heart failure and cardiac arrhythmias), and intensive care unit admission. These models were adjusted for all of the covariates in the propensity score as well as adjusted for the admitting hospital.

Statistical significance was defined as a two-tailed p-value $\leqslant 0.05$. All analyses were performed using STATA 15 (College Station, TX, USA).

\section{Results}

There was a total of 102897 patients who met the inclusion criteria. Overall, the mean age was 77.8 years (SD 7.4), 101118 (98.3\%) were male, 53252 (51.7\%) were married, 83529 (81.2\%) were white, and 6414 (6.3\%) were Hispanic. In this cohort, 23717 (23.1\%) died within 90 days.

There were 5977 patients on atypical antipsychotics prior to hospitalisation. These included: 2212 patients on quetiapine, 2205 patients on risperidone, 1375 patients on olanzapine, 361 on aripiprazole, 101 patients on ziprasidone, 46 patients on clozapine and 2 patients on paliperidone. Patients could be on more than one atypical antipsychotic during the exposure period.

\section{Propensity-matched analysis}

The propensity-matched group consisted of 11026 patients: 5513 atypical antipsychotic users and 5513 nonusers. Table 1 shows the balance between key variables after propensity matching. There were no significant differences between groups for any of the key characteristics.

In the propensity-matched cohort, 30-day mortality was $16.5 \%$ for atypical antipsychotic users versus $14.2 \%$ for nonusers $(\mathrm{p}=0.0001)$ and 90 -day mortality was $26.7 \%$ versus $23.9 \%(\mathrm{p}=0.001)$. In the regression analyses, atypical antipsychotic use was associated with increased 30-day mortality (OR1.20, 95\% CI1.11-1.31) and 90-day mortality (OR 1.19, 95\% CI 1.09-1.30). Figure 1 shows mortality and demonstrates that those who received atypical antipsychotics had significantly higher mortality than nonusers $(\mathrm{p}<0.001)$.

\section{Secondary analyses}

Table 2 shows the results of the secondary analyses. In the entire cohort, the use of atypical antipsychotics was associated with increased odds of 90-day mortality (OR 1.31, 95\% CI 1.22-1.40). For those with pre-existing psychiatric conditions, the use of atypical antipsychotics was associated with increased odds of 90-day mortality (OR 1.21, 95\% CI 1.12-1.30). For those with pre-existing cardiac conditions, atypical antipsychotics use was associated with increased odds of 90-day mortality (OR 1.22, 95\% CI 1.09-1.36). For those admitted to the intensive care unit and for those with dementia there were no statistically significant associations between atypical antipsychotic use and 90-day mortality.

\section{Discussion}

In this study, we found that there was an association between the use of atypical antipsychotics and mortality, after adjusting for potential confounders, in patients with pneumonia. While many patients hospitalised with pneumonia are receiving atypical antipsychotics, there has been a dearth of research data 
TABLE 1 Comparison of propensity-matched atypical antipsychotic users and nonusers hospitalised with pneumonia

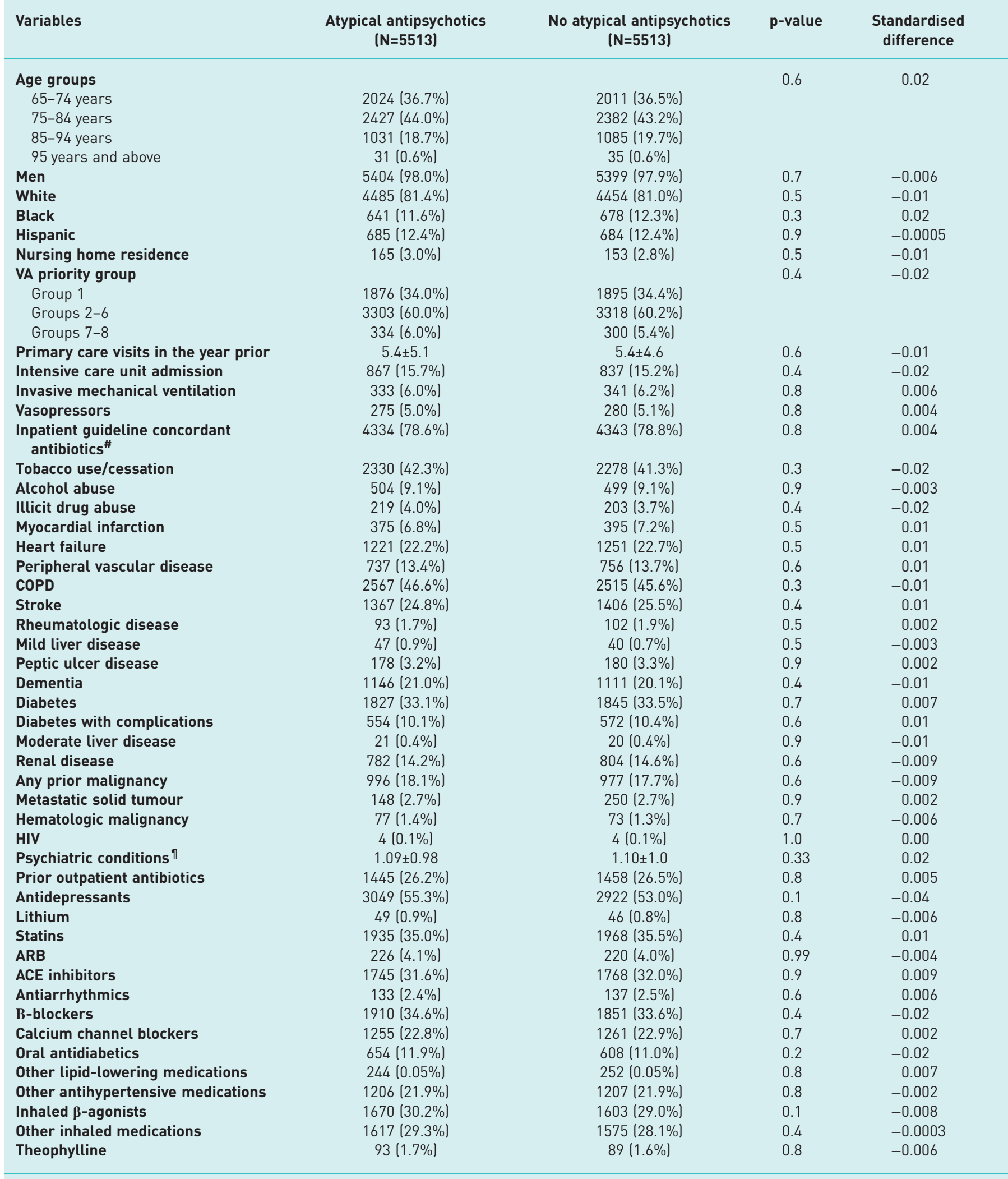

Data are presented as mean \pm SD or $n(\%)$ unless otherwise stated. VA: Dept of Veterans Affairs; ARB: angiotensin II receptor blocker; ACE: angiotensin-converting enzyme. " : concordant with 2007 American Thoracic Society/Infectious Diseases Society of America clinical practice guideline for community-acquired pneumonia [26]. ๆ.: Selim psychiatric conditions. 


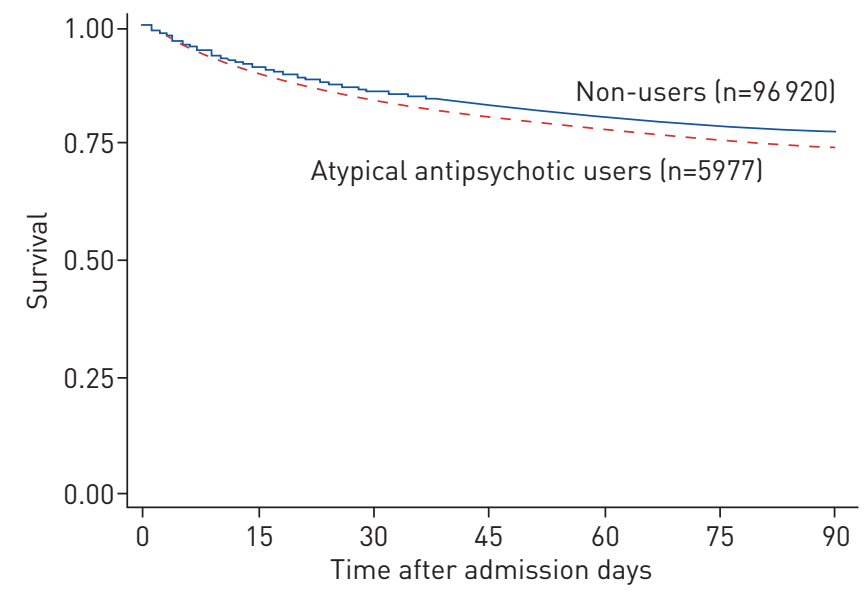

FIGURE 1 Survival curves demonstrating a statistically significant ( $p=0.001)$ association with increased mortality in those receiving atypical antipsychotics in the entire cohort.

to inform clinical decision making. In addition, most research to date has focused on the differences in outcomes between typical and atypical psychotics rather than between those who use atypical antipsychotics versus nonusers. We believe our results support the need to minimise the use of these medications for older adult hospitalised patients [22].

We hypothesise that the increased odds of mortality seen for those using atypical antipsychotics may be due to an increased QT interval arrhythmia resulting in torsades de pointes. Multiple studies [23-25] have suggested that the mechanism is a torsade de pointes associated with a prolonged QT interval, which can both lead to sudden cardiac death. Glassman et al. [24] found different risk levels of sudden cardiac death among antipsychotics, even within the same class (typical or atypical). We suggest that the increased mortality risk seen with atypical antipsychotic patients is the result of arrhythmias caused by a prolonged QT interval.

Other studies found no significant association between the use of atypical antipsychotics and mortality. Barnett et al. [5] found no increased mortality risk for patients with pneumonia taking atypical antipsychotics. The study consisted of 14057 patients hospitalised in the VA system with a primary diagnosis of pneumonia. They found no significant association between atypical antipsychotic use with in-hospital mortality of veterans (OR $1.20,95 \%$ CI $0.96-1.50$ ). We believe that our results differ as they did not control for significant confounding variables such as smoking and socioeconomic status, both of which could have significantly affected their outcomes. In addition, our study examined both in-hospital and outpatient mortality, had a larger population, and a more representative race profile.

Our study has several limitations. We studied the VA population, which may not be representative of the general population. Our sample was predominantly men, and further research is needed to examine these issues in women. Our study was restricted to those $\geqslant 65$ years of age as the source dataset was focused on that age group, so additional research is needed for other age groups. Also due to the retrospective study design and inability to examine ECGs obtained during the hospitalisation, we were unable to identify QT prolongation, torsades de pointes, or the outcome of sudden cardiac death, which we believe would be the ideal outcome for this study. That is why we focused on mortality. Unfortunately, we know of no way to adjudicate sudden cardiac death in a valid manner retrospectively. Also, for this analysis we only examined prior outpatient use of atypical antipsychotics; however, we hypothesise that most patients would have these medications continued as inpatients and after discharge. Finally, as in a nonrandomised study, we

\section{TABLE 2 Results of the adjusted multilevel regression models for secondary analyses}

\begin{tabular}{|c|c|c|}
\hline Subgroup & Patients & 90-day mortality OR $(95 \% \mathrm{CI})$ \\
\hline Entire cohort & 102976 & $1.31(1.22-1.40)$ \\
\hline Dementia & 5156 & $0.96(0.82-1.12)$ \\
\hline Psychiatric conditions ${ }^{\#}$ & 26006 & $1.21(1.12-1.30)$ \\
\hline Pre-existing cardiac conditions ${ }^{\pi}$ & 36379 & $1.22(1.09-1.36)$ \\
\hline Intensive care unit admission & 17738 & $0.96(0.82-1.12)$ \\
\hline
\end{tabular}


cannot assert causality between the exposure and outcome; however, we believe that our findings still have importance for those who care for patients with pneumonia.

In conclusion, in older adult veterans admitted with pneumonia we found an association between prior atypical antipsychotic use and mortality. This was particularly pronounced for patients with pre-existing psychiatric or cardiac conditions. We believe that physicians should be aware of this association and should use this to make informed decisions when using this family of drugs in adults over 65 years old. Further studies should be performed to better characterise the associations we have presented.

Support statement: This project was supported by grant number R01NR010828 from the National Institute of Nursing Research. E.M. Mortensen was supported by 1R24HS022418-01 from the Agency for Health Care Research and Quality. C.A. Alvarez was supported by K08DK101602 from the National Institute of Diabetes and Digestive and Kidney Diseases. Funding information for this article has been deposited with the Crossref Funder Registry.

Conflict of interest: None declared.

\section{References}

1 Jain S, Self WH, Wunderink RG, et al. Community-acquired pneumonia requiring hospitalization among U.S. adults. New Engl J Med 2015; 373: 415-427.

2 Kochanek KD, Murphy SL, Xu J, et al. Deaths: final data for 2014. Natl Vital Stat Rep 2016; 65: 1-122.

3 Trifirò G. Antipsychotic drug use and community-acquired pneumonia. Curr Infect Dis Rep 2011; 13: 262-268.

4 Knol W, van Marum RJ, Jansen PA, et al. Antipsychotic drug use and risk of pneumonia in elderly people. J Am Geriatr Soc 2008; 56: 661-666.

5 Barnett MJ, Perry PJ, Alexander B, et al. Risk of mortality associated with antipsychotic and other neuropsychiatric drugs in pneumonia patients. J Clin Psychopharmacol 2006; 26: 182-187.

6 Mortensen EM, Halm EA, Pugh MJ, et al. Association of azithromycin with mortality and cardiovascular events among older patients hospitalized with pneumonia. JAMA 2014; 311: 2199-2208.

7 Ramirez J, Aliberti S, Mirsaeidi M, et al. Acute myocardial infarction in hospitalized patients with community-acquired pneumonia. Clin Infect Dis 2008; 47: 182-187.

8 Musher DM, Rueda AM, Kaka AS, et al. The association between pneumococcal pneumonia and acute cardiac events. Clin Infect Dis 2007; 45: 158-165.

9 Corrales-Medina VF, Fatemi O, Serpa J, et al. The association between Staphylococcus aureus bacteremia and acute myocardial infarction. Scand J Infect Dis 2009; 41: 511-514.

10 Corrales-Medina VF, Serpa J, Rueda AM, et al. Acute bacterial pneumonia is associated with the occurrence of acute coronary syndromes. Medicine (Baltimore) 2009; 88: 154-159.

11 Reyes LF, Restrepo MI, Hinojosa CA, et al. Severe pneumococcal pneumonia causes acute cardiac toxicity and subsequent cardiac remodeling. Am J Respir Crit Care Med 2017; 196: 609-620.

12 Aliberti S, Tobaldini E, Giuliani F, et al. Cardiovascular autonomic alterations in hospitalized patients with community-acquired pneumonia. Respir Res 2016; 17: 98.

13 Aliberti S, Ramirez JA. Cardiac diseases complicating community-acquired pneumonia. Curr Opin Infect Dis 2014 27: 295-301.

14 Brown SH, Lincoln MJ, Groen PJ, et al. VistA-U.S. Department of Veterans Affairs national-scale HIS. Int J Med Inform 2003; 69: 135-156.

15 Murray-Thomas T, Jones ME, Patel D, et al. Risk of mortality (including sudden cardiac death) and major cardiovascular events in atypical and typical antipsychotic users: a study with the general practice research database. Cardiovasc Psychiatry Neurol 2013; 2013: 15.

16 Deyo RA, Cherkin DC, Ciol MA. Adapting a clinical comorbidity index for use with ICD-9-CM administrative databases. J Clin Epidemiol 1992; 45: 613-619.

17 Kazis LE, Miller DR, Clark J, et al. Health-related quality of life in patients served by the Department of Veterans Affairs: results from the Veterans Health Study. Arch Intern Med 1998; 158: 626-632.

18 Selim AJ, Berlowitz DR, Fincke G, et al. The health status of elderly veteran enrollees in the Veterans Health Administration. J Am Geriatr Soc 2004; 52: 1271-1276.

19 Selim AJ, Fincke G, Ren XS, et al. Comorbidity assessments based on patient report: results from the Veterans Health Study. J Ambul Care Manage 2004; 27: 281-295.

20 Sohn M-W, Arnold N, Maynard C, et al. Accuracy and completeness of mortality data in the Department of Veterans Affairs. Popul Health Metr 2006; 4: 2-2.

21 Leuven E, Sianesi B. PSMATCH2: Stata module to perform full Mahalanobis and propensity score matching, common support graphing, and covariate imbalance testing. http://ideas.repec.org/c/boc/bocode/s432001.html. Date last updated: $1 \mathrm{Feb}$ 2018. Date last accessed: July 2018.

22 McLaren KD, Marangell LB. Special considerations in the treatment of patients with bipolar disorder and medical co-morbidities. Ann Gen Hosp Psychiatry 2004; 3: 7.

23 Ray WA, Chung CP, Murray KT, et al. Atypical antipsychotic drugs and the risk of sudden cardiac death. $N$ Engl Med 2009; 360: 225-235.

24 Glassman AH, Bigger JT Jr. Antipsychotic drugs: prolonged QTc interval, torsade de pointes, and sudden death Am J Psychiatry 2001; 158: 1774-1782.

25 Curtis LH, Ostbye T, Sendersky V, et al. Prescription of QT-prolonging drugs in a cohort of about 5 million outpatients. Am J Med 2003; 114: 135-141.

26 Mandell LA, Wunderink RG, Anzueto A, et al. Infectious Diseases Society of America/American Thoracic Society consensus guidelines on the management of community-acquired pneumonia in adults. Clin Infect Dis 2007; 44: Suppl. 2, S27-S72. 